\title{
Recurrent infections associated with rare immunoglobulin isotypes deficiency
}

INSERM

\section{Source}

INSERM. (1999). Orphanet: an online rare disease and orphan drug data base. Recurrent infections associated with rare immunoglobulin isotypes deficiency. ORPHA:183675

Deficiencies in immunoglobulin (Ig) isotypes (including: isolated Ig G subclass deficiency, IgG sublcass deficiency with IgA deficiency and kappa chain deficiency) are primary immunodeficiencies that are often asymptomatic but can be characterized by recurrent, often pyogenic, sinopulmonary infections. 